\title{
Size Determination of Polystyrene Sub-Microspheres Using Transmission Spectroscopy
}

\author{
Tien Van Nguyen ${ }^{1,2} \oplus$, Linh The Pham ${ }^{3} \oplus$, Khuyen Xuan Bui ${ }^{4, *}$, Lien Ha Thi Nghiem ${ }^{5}$, \\ Nghia Trong Nguyen ${ }^{5}$, Duong Vu ${ }^{5}$, Hoa Quang Do ${ }^{5}$, Lam Dinh Vu ${ }^{6}$ \\ and Hue Minh Nguyen $2, * \mathbb{D}$ \\ 1 Department of Physics, Graduate University of Science and Technology, Vietnam Academy of Science and \\ Technology, Hanoi 10000, Vietnam; tien85ktqs@gmail.com \\ 2 Department of Physics, Le Quy Don Technical University, Hanoi 10000, Vietnam \\ 3 University of Science and Technology of Hanoi, Vietnam Academy of Science and Technology, \\ Hanoi 10000, Vietnam; linhpt8794@gmail.com \\ 4 Institute of Materials Science, Vietnam Academy of Science and Technology, Hanoi 10000, Vietnam \\ 5 Institute of Physics, Vietnam Academy of Science and Technology, Hanoi 10000, Vietnam; \\ halien@iop.vast.vn (L.H.T.N.); trongnghia@iop.vast.ac.vn (N.T.N.); duongvu@iop.vast.ac.vn (D.V.); \\ hoado@iop.vast.ac.vn (H.Q.D.) \\ 6 Graduate University of Science and Technology, Vietnam Academy of Science and Technology, Hanoi 10000, \\ Vietnam; lamvd@gust-edu.vast.vn \\ * Correspondence: khuyenbx@ims.vast.ac.vn (K.X.B.); huenm293@gmail.com (H.M.N.); \\ Tel.: +84-9061-112-521 (H.M.N.)
}

Received: 30 June 2020; Accepted: 28 July 2020; Published: 29 July 2020

check for updates

Featured Application: Polystyrene (PS) sub-microspheres have attracted much attention since they are the building blocks of many experiments, for example, in the preparation of photonics, for hybrid metal/dielectric plasmonic structures that have optical resonance in the visible/near-infrared range. Scanning electron microscopy (SEM), dynamic laser scattering (DLS), and nanoparticle tracking analysis (NTA) are widely used to determine the sizes of PS sub-microspheres. However, this equipment is not accessible for many labs, especially chemistry labs. This work demonstrates a simple method to estimate the sizes of PS sub-microspheres using a UV-vis spectrometer. This equipment is more common in labs and is routinely used to estimate the sizes of colloidal quantum dots (QDs) and metallic nanoparticles. Besides this, our proposed method does not require any curve fitting process; hence, it is applicable for researchers of other disciplines. This advantage makes the proposed method very convenient and practical for use in addition to other methods.

\begin{abstract}
Nano/micro polystyrene (PS) beads have found many applications in different fields spanning from drug delivery, bio-diagnostics, and hybrid plasmonics to advanced photonics. The sizes of the PS beads are an important parameter, especially in plasmonic and photonic experiments. In this work, we demonstrate a quick and straightforward method to estimate the diameters of sub-microspheres $(0.2 \mu \mathrm{m}$ to $0.8 \mu \mathrm{m})$ using the transmission spectra of a close-packed monolayer of polystyrene beads on glass or quartz substrates. Experimental transmission spectra of the PS monolayers were verified against finite-difference time-domain (FDTD) simulation and showed good agreement. The effects of the substrates on the transmission spectra and, hence, the accuracy of the method were also studied by simulation, which showed that common transparent substrates only cause minor deviation of the PS bead sizes calculated by the proposed method.
\end{abstract}

Keywords: photonic crystals; polystyrene sub-microspheres; finite-difference time-domain (FDTD) 


\section{Introduction}

Monodisperse latex particles have a curious history of being one of the first products made in space on the Space Shuttle missions [1]. The preparation of polystyrene (PS) microspheres of uniform size was developed over half a century ago, early in the 1950s [2]. However, in recent years, one can still find reports on new synthesis techniques of monodisperse, controlled-size, surface-functionalized, fluorescent PS microspheres [3-5]. This attention to PS microspheres is, on the one hand, due to their broad scope of applications, starting from well-known applications, namely, as calibration standards, for flow cytometry, for fluorescence microscopy, as support materials for biochemical analysis, and for drug delivery [6,7], to more recently reported uses in the fabrication of new materials, such as Pickering emulsions [8] and capsules with nonhomogenous shells [9], and in the assembly of 1D granular structures [10]. On the other hand, possessing controllable size and ideal spherical shapes, PS microspheres themselves have been model systems for studying new physical mechanisms of particle-covered drops in electric fields [11], and they have been key experimental objects for many research works in the field of photonics. Interesting optical phenomena have been observed with microspheres, such as micro-lasers [12] and super focusing of light via photonic nanojets [13]. Periodically ordered structures of self-assembled PS microspheres have also attracted much attention. These structures could serve as ideal platforms to study the optical properties of 2D and 3D photonic crystals $[3,14,15]$ and as a tool in a process called nanosphere lithography for the controlled manufacture of ordered metal-dielectric hybrid nanostructures [16,17].

The sizes of the PS microspheres are one of the main tuning parameters in the experiments mentioned above. The diameters of micrometer-sized PS beads can be easily determined by an optical microscope using the array sizing method [18]. However, sophisticated equipment such as a scanning electron microscope (SEM), dynamic laser scattering (DLS) equipment, and nanoparticle tracking analysis (NTA) equipment [19] are needed to measure the diameters of PS sub-microspheres. It is tempting to exploit UV-vis spectroscopy, which is more common in labs and is routinely used to estimate the sizes of colloidal quantum dots (QDs) [20] and metallic nanoparticles [21,22], for the size determination of PS sub-micrometer beads.

In principle, the scattering extinction coefficient as a function of wavelength can be measured using a UV-vis spectrometer, and the corresponding scattering cross section (diameter) of the particles can then be determined. Zheng et al. systematically studied the wavelength, size, and angle dependences of light scattering of $20 \mathrm{~nm}$ to $85 \mu \mathrm{m}$ diameter PS microspheres in water. The experimental results were verified against Rayleigh, Mie, and Tyndall's light scattering theories and were in good agreement with the theoretical predictions [23]. Optical transmission spectra in the 400-800 $\mathrm{nm}$ range for samples of sub-monolayers of $0.5 \mu \mathrm{m}$ to $1 \mu \mathrm{m}$ diameter PS microspheres reported by Stanley's group were also dominated by light scattering and were microsphere-size-dependent [24]. However, it is not easy to retrieve the sizes of the microspheres from these spectra since diameters can only be determined through complicated curve fitting procedures in the framework of light scattering theories.

The transmission spectra of 3D and 2D photonic crystals based on microspheres have been thoroughly studied both theoretically and experimentally [14,25-27]. On the spectra, new, distinct features related to the photonic bandgap and optical modes appeared. The spectral peak positions of these features strongly depend on the sizes of the microspheres. In this paper, we demonstrate that this property could be employed to determine the dimensions of polystyrene sub-microspheres. It is also worth mentioning that fabrication of the PS microsphere 2D photonic crystals is simple, this proposed method does not require high-quality 2D photonic samples, and microsphere sizes can be estimated using a simple formula instead of curve fitting. This simplicity makes the method very practical. The experimental results were compared with simulations via the software CST- a finite-difference time-domain (FDTD) electromagnetic field solver. Good agreement between the experimental and simulated transmission spectra further confirmed both the experiment and the adequacy of the theoretical model. The influence of the substrate refractive indexes on the peak 
positions and its consequences on PS bead size estimation were also studied. The analysis showed that common transparent substrates could cause minor shifts and broadening of the peak.

\section{Materials and Methods}

Monodispersed PS microspheres were prepared in aqueous alcohol systems following procedures previously developed by Zhang et al. [28]. Briefly, styrene was emulsion polymerized in a solution of sodium lauryl sulfate, potassium persulfate, and aqueous ethanol, which serve as the emulsifier, initiator, and dispersion medium, respectively. Controlled amounts of sodium lauryl sulfate and potassium persulfate were dissolved in $7 \mathrm{~mL}$ aqueous ethanol (volumetric ratio of deionised (DI) water to ethanol was $2 / 5, v / v$ ). The solution was kept in a small glass vial, thoroughly mixed, and temperature controlled by a magnetic stirrer/hot plate. A measured amount of styrene was injected into the solution, and then the whole system was quickly heated up to $70^{\circ} \mathrm{C}$ and kept constant at this temperature for polymerization for $6 \mathrm{~h}$. After the polymerization reaction was completed, the PS bead dispersion was centrifuged and washed with ethanol three times to remove the coagulate, incompletely reacted styrene, and soluble impurities. PS beads of different sizes were synthesized. SEM image analysis of 100 PS beads of each size showed that the prepared PS beads had a spherical shape and size distribution deviation of less than $5 \%$. PS beads with diameters of $212 \pm 3,477 \pm 7,574 \pm 10,684 \pm 20$, and $812 \pm 24 \mathrm{~nm}$ were used to test the proposed method.

The close-packed monolayer of the PS microspheres was trapped and self-assembled on the air/water interface, then transferred onto clean glass, quartz, or silicon substrates by the fishing technique. This approach is an undemanding, high-yield method to prepare a relatively high-quality, large-area, close-packed monolayer of PS microspheres [29]. After synthesis, the solution containing PS beads was centrifuged and re-dispersed in aqueous ethanol $(1 / 1, v / v)$ to $10 \% w / v$ concentration. The prepared solution was used as such to assemble monolayers of PS beads. This solution was slowly dispensed using a pipette onto a clean glass slide placed against the wall of a glass container half-filled with DI water. The glass slide made an angle of $45^{\circ}$ with the wall of the container. PS beads went down the inclined plane; a small part of them sank in the water, while the rest became trapped at the interface, spread out on the water surface, and formed a monolayer of PS beads. To further consolidate the particles, a small amount of $2 \%$ SDS (sodium dodecyl sulfate) was added to change the water surface tension. This monolayer was then transferred onto clean $1 \times 2.5 \mathrm{~cm}^{2}$ glass, quartz, or silicon substrates for transmission spectra and SEM measurements. Figure 1 shows SEM images of the self-assembled monolayer of PS beads mentioned above. Some of the area of the film-shown in the inset of Figure 1d, for example-is close-packed and has the symmetry of a hexagonal lattice. There are still holes and boundaries in the films and no long-range order. However, one of the advantages of this method is that it is not necessary to have perfect 2D crystalline structures.
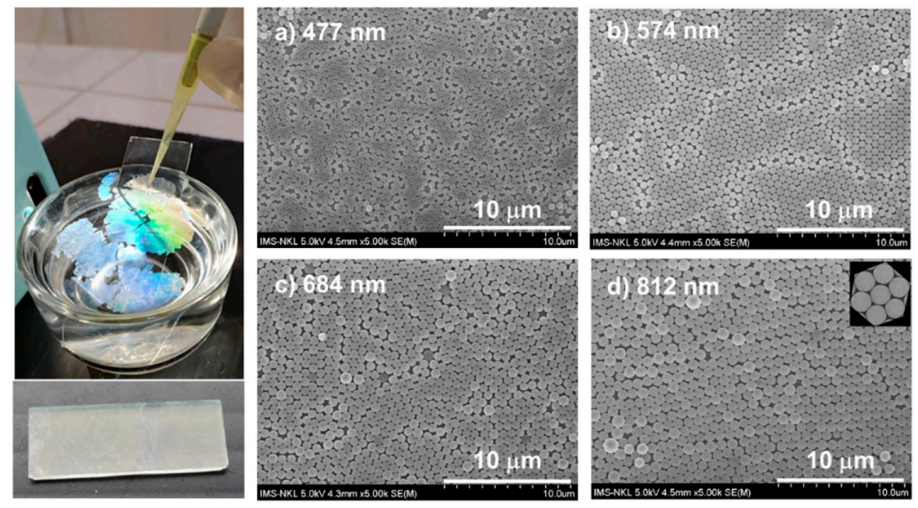

Figure 1. Trapping a monolayer of polystyrene (PS) sub-microspheres on the water/air interface (left panel), and SEM images of the close-packed monolayers of PS beads with diameters of (a) $477 \mathrm{~nm}$, (b) $574 \mathrm{~nm}$, (c) $684 \mathrm{~nm}$, and (d) $812 \mathrm{~nm}$ after transferring them onto substrates. 
For simulations, we employed the finite-difference time-domain (FDTD) solver in CST Microwave Studio software to simulate the transmission spectra of the structures. A model consisting of a monolayer of hexagonal close-packed dielectric microspheres with dielectric constant $\varepsilon_{\text {sphere }}$ on a dielectric substrate with dielectric constant $\varepsilon_{\text {sub }}$ was studied. As shown in Figure 2, the first layer of the unit cell is a sphere of diameter $d$ in contact with four quarters of a sphere at each corner, and the second layer is a dielectric substrate of thickness $t_{\mathrm{d}}$. A periodic boundary condition was applied. The period of unit cell structure is $a_{\mathrm{x}}=d$ and $a_{\mathrm{y}}=\sqrt{3} d$ in the $x$ and $y$ directions, correspondingly. In order to accelerate the computational time, the thickness $t_{\mathrm{d}}$ of the substrate was set to be $t_{\mathrm{d}}=15,000 \mathrm{~nm}$ in the simulation, instead of the actual thickness of the glass substrates. A series of parameter pre-screening tests showed that thicker $t_{\mathrm{d}}\left(t_{\mathrm{d}}>15,000 \mathrm{~nm}\right)$ has a negligible effect on the simulated transmission spectra. The dielectric constants of the spheres and substrate were chosen to be $\varepsilon_{\text {sphere }}=2.25\left(n_{\text {sphere }}=1.5\right)$ and $\varepsilon_{\text {sub }}=2.5\left(n_{\text {sub }}=1.58\right)$, respectively. These dielectric constant values are close to those of polystyrene and glass in the visible range [30,31]. In the model, the PS microspheres and dielectric substrate were considered nonabsorbing, with the imaginary part $\varepsilon^{\prime \prime}$ of the dielectric constants equal to zero. The incident electromagnetic wave is perpendicular to the surface of the structure. The electric and magnetic components of the wave are along the $\mathbf{y}$-axis and $\mathbf{x}$-axis, correspondingly. Two transmitters and receivers are located on the sides of the structure along the $\mathbf{z}$-axis to measure the transmission scattering parameters $S_{21}(\omega)$ of the electromagnetic wave when it interacts with the PS/glass medium. Then, transmittance $(T)$ would be obtained by $T(\omega)=\left|S_{21}(\omega)\right|$.
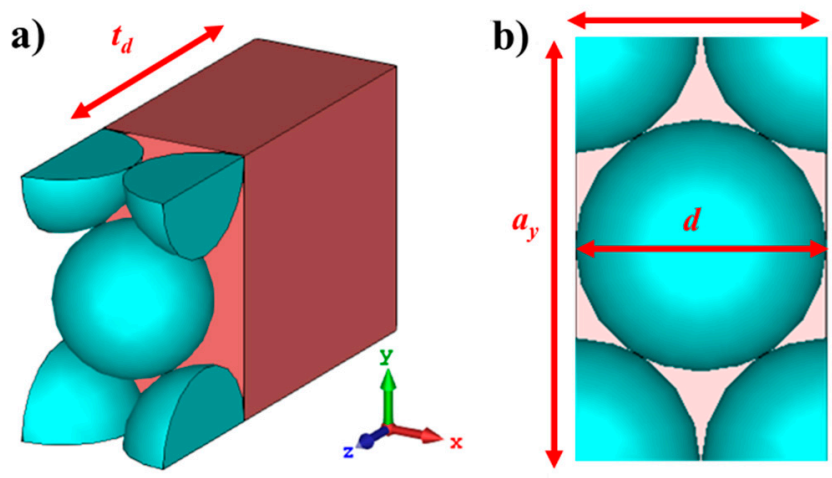

Figure 2. The layout of the computational unit cell of a close-packed hexagonal monolayer of dielectric spheres on a dielectric substrate: (a) perspective view and (b) top view.

\section{Results}

\subsection{Experimental Results}

Figure 3a shows the transmission spectra of the $574 \mathrm{~nm}$ PS beads suspended in solution, as a sub-monolayer, and as a multilayer (dotted blue, dashed brown, and dash-dotted red lines, respectively), and that of a close-packed monolayer of the PS beads (solid black line). While the spectra presented as broken lines are rather smooth and do not have distinct features, the solid black line has apparent dips. Moreover, the positions of the first dips in the transmission spectra shift monotonically and linearly toward longer wavelengths as the size of the beads increases (Figure 3b). The ideal close-packed monolayer of the spheres is arrayed in a hexagonal lattice with lattice constant $d$. Theoretical works showed that for the case of a free-standing 2D hexagonal lattice of PS beads $\left(\varepsilon_{\text {sphere }}=2.56\right)$, dips in the transmission spectra arise at the spectral positions where parameter $Z=(\sqrt{3} d) / 2 \lambda$ satisfies condition $Z=0.71,0.85,1.00,1.34$, or 1.55 [27]. Our simulation for the case of a hexagonal lattice of PS beads $\left(\varepsilon_{\text {sphere }}=2.25\right)$ on a glass substrate $\left(\varepsilon_{\text {sub }}=2.5\right)$ shows that the most pronounced dip (the first dip from the left) in the transmission spectra in Figure $3 b$ corresponds to $Z=0.7$. 

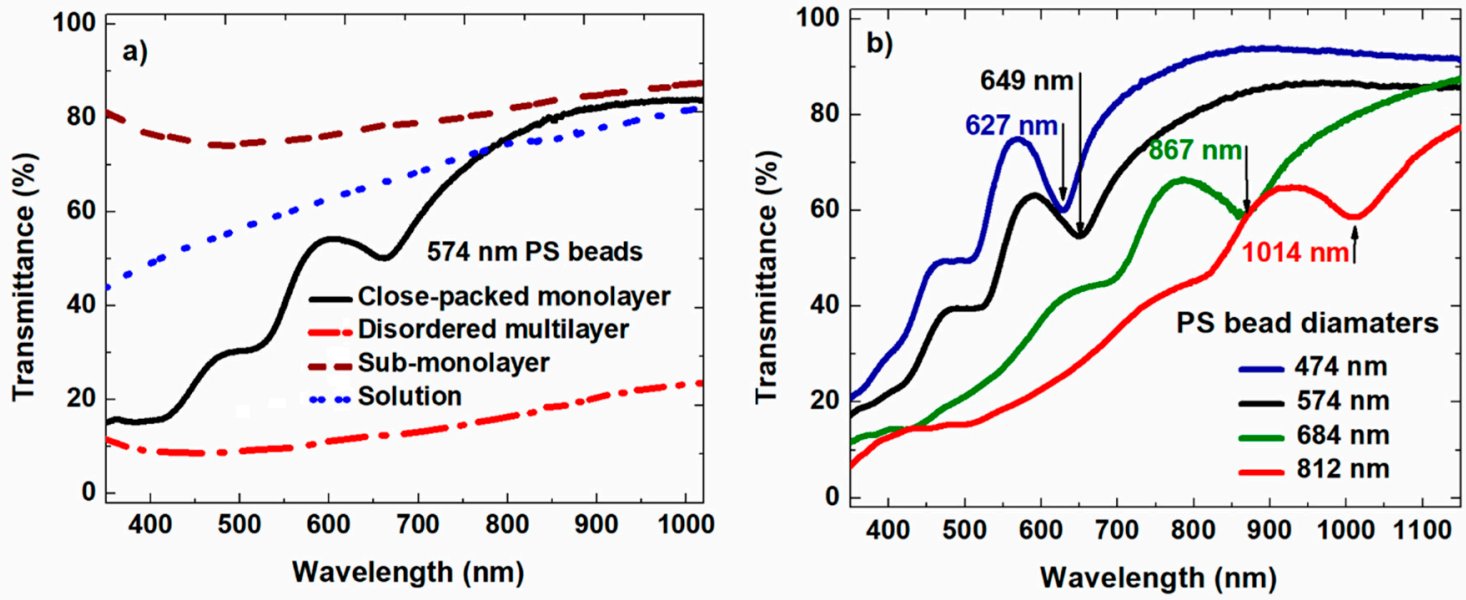

Figure 3. Measured transmission spectra of (a) $574 \mathrm{~nm}$ PS beads suspended in solution, as a sub-monolayer, as a multilayer, and as a close-packed monolayer (dotted blue, dashed brown, dash-dotted red, and solid black lines, respectively) and of (b) self-assembled films of PS beads with diameters of $474,574,684$, and $812 \mathrm{~nm}$. The transmission dips in the spectra shift to longer wavelengths as the size of the beads increases.

\subsection{Simulation}

Figure 4a shows the simulated transmission spectra when the dielectric constant of the substrate was varied from 1.5 to 3 . This range of dielectric constants covers most typical glass substrates. When the dielectric constant of the substrate varies from 1.5 to 3 , the position of the first peak $(Z=0.7)$ stays nearly unchanged, while the second peak $(Z=0.85)$ undergoes a slight blue-shift $(15 \mathrm{~nm})$.
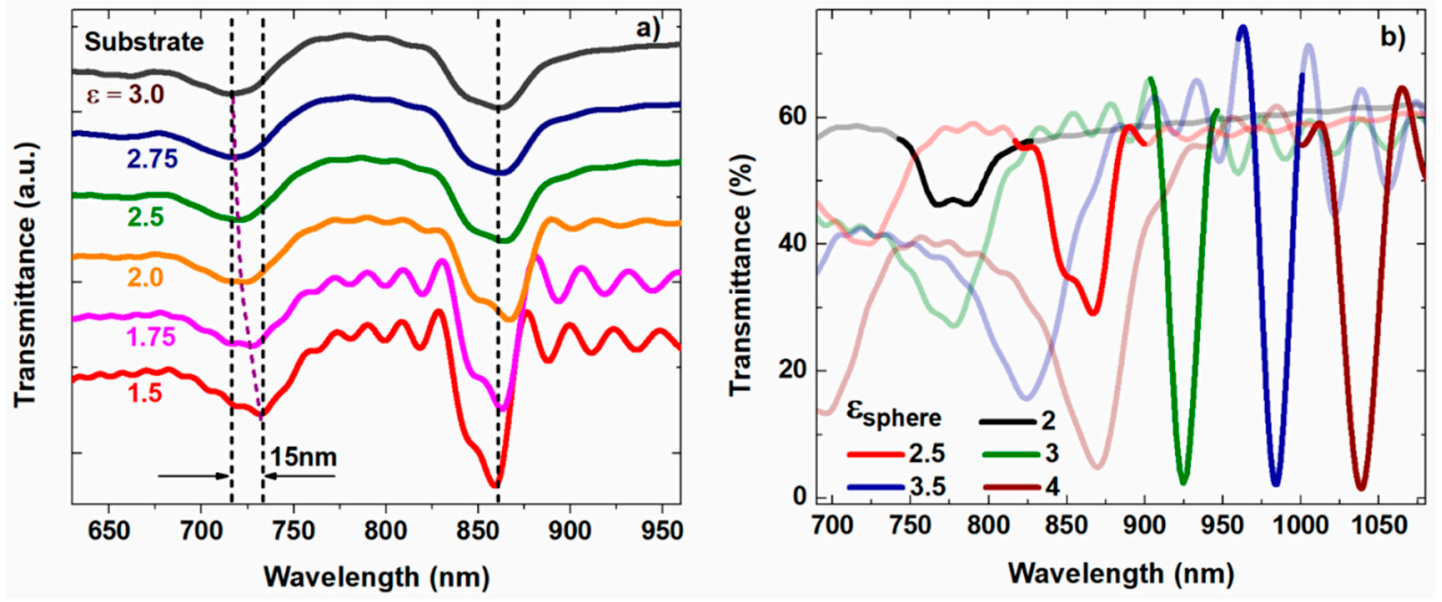

Figure 4. Simulated transmission spectra of a close-packed monolayer of $701 \mathrm{~nm}$ PS beads on a dielectric substrate (a) fixing $\varepsilon_{\text {sphere }}=2.25$ while the dielectric constant of the substrate varies in the range of 1.5 to 3 and (b) keeping $\varepsilon_{\text {sub }}=2.5$ while the dielectric constant of the dielectric spheres changes from 2 to 4 .

\section{Discussion}

The simulated transmission spectra of a close-packed monolayer of PS beads on a glass substrate in comparison with the experimental data are shown in Figure 5. The simulated curves have dips corresponding to the resonance frequencies and lay under the experimental curves. As mentioned earlier, the samples are not ideal 2D photonic crystals; therefore, they have smaller fill factors which make the measured transmittance higher. Moreover, due to imperfection in the experimental samples, most of the dips are also smeared out in the measured transmission curves. Only the first $\operatorname{dip}(Z=0.7)$ is the most noticeable. 


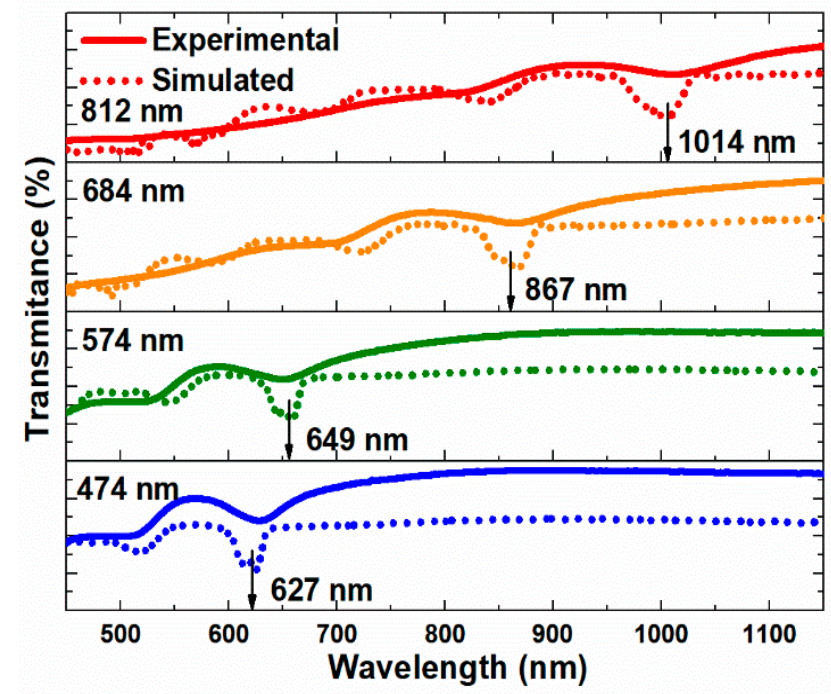

Figure 5. Transmittance spectra of a close-packed monolayer of PS beads; solid lines indicate experimental results and dotted lines indicate simulated results. The parameters for the simulations were $\varepsilon_{\text {sphere }}=2.25\left(n_{\text {sphere }}=1.5\right)$ and $\varepsilon_{\text {sub }}=2.5\left(n_{\text {sphere }}=1.58\right)$, diameters of the PS microspheres: $507,525,701$, and $820 \mathrm{~nm}$.

The spectral position of this dip is used to estimate the diameter of the PS sub-microspheres by a simple formula:

$$
d=\frac{2 \times Z \times \lambda_{1^{s t}} \text { dip }}{\sqrt{3}}
$$

where $d$ and $\lambda_{1^{s t}}$ dip (corresponding to $Z=0.7$ ) are the diameter of the PS beads and the spectral position of the first dip in the transmission spectrum, respectively.

In Figure 6, the diameters of the PS beads are plotted against the spectral positions of the first dips appearing in the transmission spectra of close-packed monolayers of the PS beads. These data points were well fitted by a simple linear function with a slope of 0.799 . The $Z$ parameter calculated from the slope of the plot was $Z=0.69$, which agrees very well with the theoretical value $Z=0.7$. This result, first of all, confirms the validity of the physical model. Hence, it allows us to use Formula (1) for the size estimation of PS sub-microspheres.

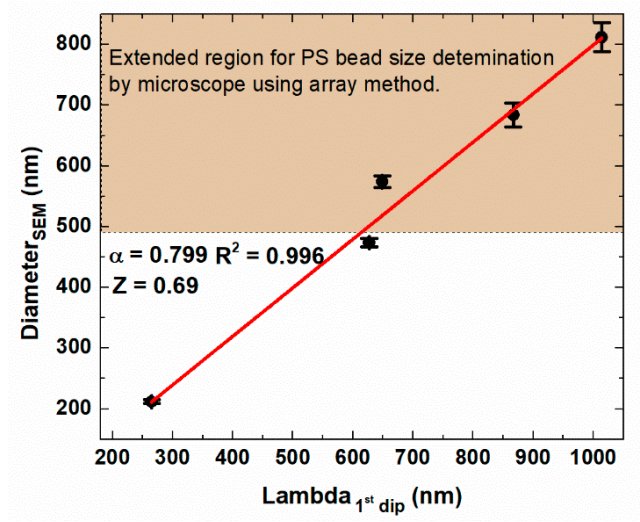

Figure 6. The diameters of the PS beads plotted against the spectral positions of the first dips. The $Z$ parameter retrieved from the slope of the plot was $Z=0.69$, which agrees well with the theoretical value $Z=0.7$ used for finite-difference time-domain (FDTD) simulation.

This technique works well with PS beads of diameter as small as $200 \mathrm{~nm}$. It further extends the capability of the optical method for the size determination of PS beads to the sub-micrometer range [32]. 
Unfortunately, when the size of the PS beads reaches the nanometer region, the spectral position of the first dip in the transmission spectrum falls in the deep UV range and cannot be measured by the detectors of common commercial UV-vis spectrometers.

Glass substrates can be used to determine the sizes of PS beads with diameters in the upper sub-micrometer range. However, quartz substrates are necessary for smaller PS bead size determination. The dielectric constant of the substrate can affect the spectra in general and the spectral position of the first dip specifically. While the latter directly defines the estimated size of the PS beads, the simulation showed that the first peak $(Z=0.7)$ is insensitive to the refractive index of the substrate (Figure 4a). This makes the proposed method convenient since the choice of substrate materials is relaxed. In consequence, estimation based on this dip would be more accurate.

This method could also be utilized for the size determination of nano/microspheres made of other materials. Figure $4 \mathrm{~b}$ presents the simulated transmission spectra of different monolayers of dielectric spheres. When the dielectric constant of the dielectric spheres spans from 2 to 4 , the spectral position of the first dip shifts to the right. The new values of $Z$ for microspheres of different dielectric constants can be recalculated by simulation using the new spectral position of the first dip and the size of the microspheres used to simulate the transmission spectrum.

\section{Conclusions}

We demonstrated herein a quick and straightforward method to estimate the sizes of PS sub-microspheres via their transmission spectra. This method was verified on PS beads of different sizes that were prepared by emulsion polymerization. The diameters of the PS beads defined by this method and measured by SEM are in good agreement. The experimental transmission spectra fit well with the results simulated by FDTD simulation. These results, on the one hand, give a firm theoretical foundation for the proposed method; on the other hand, they help us to understand the effects of common glass substrates on the accuracy of the size estimation. The analysis showed that the influence of the glass substrates is negligible. These advantages make the proposed method convenient and practical for use in addition to other well-known routines for PS size determination.

Author Contributions: Conceptualization, H.M.N. and K.X.B.; methodology, T.V.N., L.T.P., L.H.T.N. and N.T.N.; formal analysis, H.M.N., D.V., L.T.P.; investigation, T.V.N.; resources, L.H.T.N.; writing-original draft preparation, H.M.N., D.V., and T.V.N.; writing-review and editing, K.X.B., H.Q.D. and L.D.V.; visualization, H.M.N., L.T.P. and D.V.; supervision, H.M.N.; funding acquisition, H.M.N. and K.X.B. All authors have read and agreed to the published version of the manuscript.

Funding: This research was supported by Vietnam National Foundation for Science and Technology Development (NAFOSTED) under grant No. 103.03-2016.73; and by the Institute of Materials Science, Vietnam Academy of Science and Technology under Grant No. HTCBT.05/20-20.

Conflicts of Interest: The authors declare no conflict of interest. The funders had no role in the design of the study; in the collection, analyses, or interpretation of data; in the writing of the manuscript; or in the decision to publish the results.

\section{References}

1. Vanderhoff, J.W.; El-Aasser, M.S.; Kornfeld, D.M.; Micale, F.J.; Sudol, E.D.; Tseng, C.-M.; Sheu, H.-R. The First Products Made in Space: Monodisperse Latex Particles. MRS Online Proc. Libr. Arch. 1986, 87, 213. [CrossRef]

2. Alfrey, T.; Bradford, E.B.; Vanderhoff, J.W.; Oster, G. Optical Properties of Uniform Particle-Size Latexes*. J. Opt. Soc. Am. 1954, 44, 603. [CrossRef]

3. Shao, J.; Zhang, Y.; Fu, G.; Zhou, L.; Fan, Q. Preparation of monodispersed polystyrene microspheres and self-assembly of photonic crystals for structural colors on polyester fabrics. J. Text. Inst. 2014, 105, 938-943. [CrossRef]

4. Sekerak, N.M.; Hutchins, K.M.; Luo, B.; Kang, J.G.; Braun, P.V.; Chen, Q.; Moore, J.S. Size control of cross-linked carboxy-functionalized polystyrene particles: Four orders of magnitude of dimensional versatility. Eur. Polym. J. 2018, 101, 202-210. [CrossRef] 
5. Lee, J.; Ha, J.U.; Choe, S.; Lee, C.S.; Shim, S.E. Synthesis of highly monodisperse polystyrene microspheres via dispersion polymerization using an amphoteric initiator. J. Colloid Interface Sci. 2006, 298, 663-671. [CrossRef] [PubMed]

6. Chandler, W.L.; Yeung, W.; Tait, J.F. A new microparticle size calibration standard for use in measuring smaller microparticles using a new flow cytometer. J. Thromb. Haemost. 2011, 9, 1216-1224. [CrossRef]

7. Kaur, H.; Kumar, S.; Kukkar, D.; Kaur, I.; Singh, K.; Bharadwaj, L.M. Transportation of Drug-(Polystyrene Bead) Conjugate by Actomyosin Motor System. J. Biomed. Nanotechnol. 2010, 6, 279-286. [CrossRef]

8. Rozynek, Z.; Bielas, R.; Józefczak, A. Efficient formation of oil-in-oil Pickering emulsions with narrow size distributions by using electric fields. Soft Matter 2018, 14, 5140-5149. [CrossRef]

9. Kubiak, T.; Banaszak, J.; Józefczak, A.; Rozynek, Z. Direction-Specific Release from Capsules with Homogeneous or Janus Shells Using an Ultrasound Approach. ACS Appl. Mater. Interfaces 2020, 12, 15810-15822. [CrossRef]

10. Mikkelsen, A.; Kertmen, A.; Khobaib, K.; Rajňák, M.; Kurimský, J.; Rozynek, Z. Assembly of 1D Granular Structures from Sulfonated Polystyrene Microparticles. Materials 2017, 10, 1212. [CrossRef]

11. Mikkelsen, A.; Khobaib, K.; Eriksen, F.K.; Måløy, K.J.; Rozynek, Z. Particle-covered drops in electric fields: Drop deformation and surface particle organization. Soft Matter 2018, 14, 5442-5451. [CrossRef] [PubMed]

12. Fernandez-Bravo, A.; Yao, K.; Barnard, E.S.; Borys, N.J.; Levy, E.S.; Tian, B.; Tajon, C.A.; Moretti, L.; Altoe, M.V.; Aloni, S.; et al. Continuous-wave upconverting nanoparticle microlasers. Nat. Nanotechnol. 2018, 13, 572-577. [CrossRef]

13. Heifetz, A.; Kong, S.-C.; Sahakian, A.V.; Taflove, A.; Backman, V. Photonic Nanojets. J. Comput. Theor. Nanosci. 2009, 6, 1979-1992. [CrossRef]

14. Ma, X.; Yan, Z. The size influence of silica microspheres on photonic band gap of photonic crystals. Int. J. Mod. Phys. B 2007, 21, 2761-2768. [CrossRef]

15. Schweizer, T. From polystyrene beads to photonic devices for telecommunication. SPIE Newsroom. 2008. [CrossRef]

16. Miller, E.N.; Palm, D.C.; de Silva, D.; Parbatani, A.; Meyers, A.R.; Williams, D.L.; Thompson, D.E. Microsphere lithography on hydrophobic surfaces for generating gold films that exhibit infrared localized surface plasmon resonances. J. Phys. Chem. B 2013, 117, 15313-15318. [CrossRef] [PubMed]

17. Domonkos, M.; Varga, M.; Ondič, L.; Gajdošová, L.; Kromka, A. Microsphere lithography for scalable polycrystalline diamond-based near-infrared photonic crystals fabrication. Mater. Des. 2018, 139, 363-371. [CrossRef]

18. Mulholland, G.W.; Hartman, A.W.; Hembree, G.G. Development of a One-Micrometer-Diameter Particle Size Standard Reference Material. J. Res. Natl. Bur. Stand. 1985, 90, 3-26. [CrossRef]

19. Filipe, V.; Hawe, A.; Jiskoot, W. Critical evaluation of nanoparticle tracking analysis (NTA) by NanoSight for the measurement of nanoparticles and protein aggregates. Pharm. Res. 2010, 27, 796-810. [CrossRef]

20. Yu, W.W.; Qu, L.; Guo, W.; Peng, X. Experimental determination of the extinction coefficient of CdTe, CdSe, and CdS nanocrystals. Chem. Mater. 2003, 15, 2854-2860. [CrossRef]

21. Baset, S.; Akbari, H.; Zeynali, H.; Shafie, M. Size measurement of metal and semiconductor nanoparticles via UV-Vis absorption spectra. Dig. J. Nanomater. Biostructures (DJNB) 2011, 6, 709-716.

22. Haiss, W.; Thanh, N.T.K.; Aveyard, J.; Fernig, D.G. Determination of size and concentration of gold nanoparticles from UV-Vis spectra. Anal. Chem. 2007, 79, 4215-4221. [CrossRef] [PubMed]

23. He, G.S.; Qin, H.Y.; Zheng, Q. Rayleigh, Mie, and Tyndall scatterings of polystyrene microspheres in water: Wavelength, size, and angle dependences. J. Appl. Phys. 2009, 105, 023110. [CrossRef]

24. Guillaumée, M.; Liley, M.; Pugin, R.; Stanley, R.P. Scattering of light by a single layer of randomly packed dielectric microspheres giving color effects in transmission. Opt. Express 2008, 16, 1440. [CrossRef] [PubMed]

25. Farcău, C.; Vinteler, E.; Aştilean, S. Experimental and theoretical investigation of optical properties of colloidal photonic crystal films. J. Optoelectron. Adv. Mater. 2008, 10, 3165-3168.

26. Kurokawa, Y.; Miyazaki, H.; Jimba, Y. Light scattering from a monolayer of periodically arrayed dielectric spheres on dielectric substrates. Phys. Rev. B 2002, 65, 201102. [CrossRef]

27. Miyazaki, H.; Ohtaka, K. Near-field images of a monolayer of periodically arrayed dielectric spheres. Phys. Rev. B 1998, 58, 6920-6937. [CrossRef]

28. Zhang, J.; Chen, Z.; Wang, Z.; Zhang, W.; Ming, N. Preparation of monodisperse polystyrene spheres in aqueous alcohol system. Mater. Lett. 2003, 57, 4466-4470. [CrossRef] 
29. Rybczynski, J.; Ebels, U.; Giersig, M. Large-scale, 2D arrays of magnetic nanoparticles. Colloids Surf. A Physicochem. Eng. Asp. 2003, 219, 1-6. [CrossRef]

30. Malitson, I.H. Interspecimen Comparison of the Refractive Index of Fused Silica. J. Opt. Soc. Am. 1965, 55, 1205. [CrossRef]

31. Sultanova, N.; Kasarova, S.; Nikolov, I. Dispersion Properties of Optical Polymers. Acta Phys. Pol. -Ser. A Gen. Phys. 2009, 116, 585-587. [CrossRef]

32. Duke, S.D.; Layendecker, E.B. Improved array method for size calibration of monodisperse spherical particles by optical microscope. Part. Sci. Technol. 1989, 7, 209-216. [CrossRef]

(C) 2020 by the authors. Licensee MDPI, Basel, Switzerland. This article is an open access article distributed under the terms and conditions of the Creative Commons Attribution (CC BY) license (http://creativecommons.org/licenses/by/4.0/). 